\title{
The effect of the Corona measures in Germany Pre-Analysis Plan
}

\author{
Michael Ahlheim* Kai Konrad ${ }^{\dagger} \quad$ Lisa Windsteiger ${ }^{\ddagger}$
}

April 9, 2020

JEL classification codes: D78, C99

Keywords: COVID-19, subjective well-being, survey experiment Declaration of interests: None

\section{Introduction}

On March 22, 2020, the German federal government imposed a variety of restrictive measures to curb the spread of the Corona-Virus. Among these measures were severe restrictions on social contacts, the closure of non-essential shops, restaurants and bars, the cancellation of events of any size, the closure of schools and day care facilities, and severe restrictions of visits to old people's homes and hospitals. We use an online survey to investigate how the German population was affected by these measures in terms of their subjective well-being, whether they approve of the measures and whether they want to continue those measures beyond April 20. Additionally, we want to elicit how much they would be willing to pay for an abolition of the measures. Furthermore, we want to elicit how these effects vary with personal characteristics such as psychological reactance and locus of control, but also socioeconomic variables such as income and type of employment (self-employed, working in the public sector,..)

\footnotetext{
*University of Hohenheim ahlheim@uni-hohenheim.de

$\dagger$ Max Planck Institute for Tax Law and Public Finance. kai.konrad@tax.mpg.de

${ }_{\ddagger}^{\ddagger}$ Max Planck Institute for Tax Law and Public Finance. lisa.windsteiger@tax.mpg.de
} 
Finally, we want to explore whether the Corona Crisis and the imposed restrictive measures have created a (latent) generational conflict: As the virus has turned out to be much more dangerous for older generations, it can be argued that the restrictive measures protect (and thus benefit) them most, while younger (working-age) generations are those harmed most economically (because they lose their jobs and businesses etc.). We want to explore whether (experimentally) making this generational conflict more salient affects people's support for a continuation/abolition of those restrictive measures.

\section{$2 \quad$ Experimental strategy}

The experimental component of our research design consists of the random provision of information about the case fatality rates (CFRs) related to COVID-19 in China to subgroups of a representative German online survey panel. We aim at systematically varying whether the respondents receive information on the average CFR vs. information on how the rate varies by age group. The numbers come from a study conducted in China, where the Corona-Virus broke out first. ${ }^{1}$

\subsection{Information conditions}

We randomly assign the respondents to one of three information conditions. To provide a clear baseline, we include a "neutral condition" in which respondents will read a neutral sentence only referring to the fact that the government introduced measures to combat the spread of COVID-19. The other conditions (also include that sentence and) vary whether respondents are informed about:

- the average estimated case fatality rate of COVID-19 (Condition Average),

- the CFR by age group, indicating a clear increase of fatalities for higher age groups (Condition Age),

The experimental information is conveyed to the respondents by means of video clips which they visualise in the course of the survey. Stills of the clips are provided in Figures 1 to 3.

Each of the videos begins with a statement referring to the fact that the government introduced measures to combat the spread of COVID-19. Only the video for the Neutral condition then continues immediately with an invitation for the respondent

\footnotetext{
${ }^{1}$ http://weekly.chinacdc.cn/en/article/id/e53946e2-c6c4-41e9-9a9b-fea8db1a8f51
} 
to proceed with the survey. All the other videos inform our respondents of the estimated case fatality rate of COVID-19 as described above.

Condition Neutral The information provided in the Neutral condition only generically refers to the fact that the government introduced measures to combat the spread of COVID-19 without mentioning estimated case fatality rates.

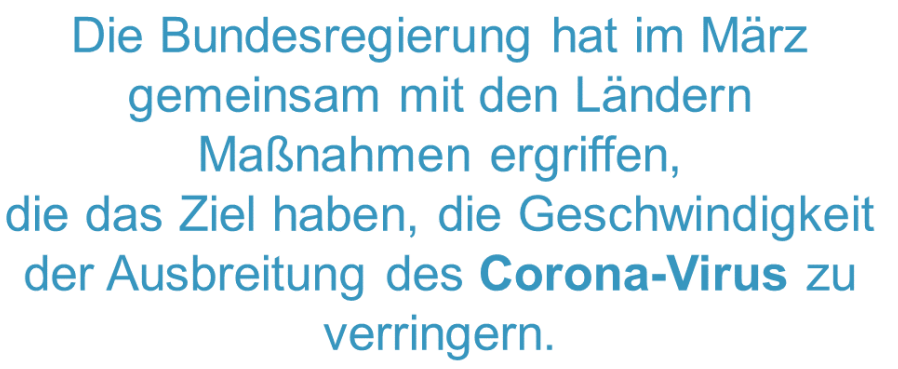

Figure 1: Information provided in the Neutral condition 
Condition Average Figure 2 displays the information given to the respondents assigned to Condition HH. This group of respondents receive information about the fact that the estimated average case fatality rate of COVID-19 in China was 2.3\%.

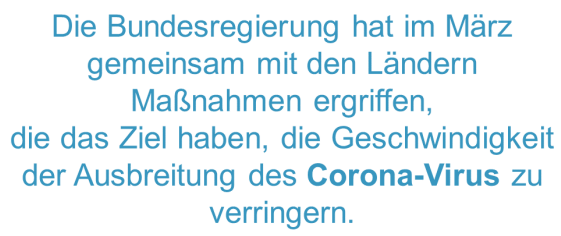
gemeinsam mit den Ländern Maßnahmen ergriffen, die das Ziel haben, die Geschwindigkeit der Ausbreitung des Corona-Virus zu verringern.

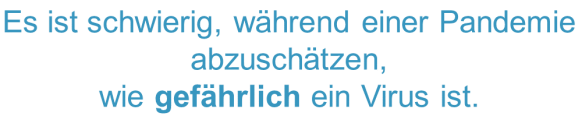

(a)

Eine Studie für China zeigt, dass dort etwa 2,3\% der Personen, die mit Corona infiziert waren, im Krankheitsverlauf gestorben sind.
4

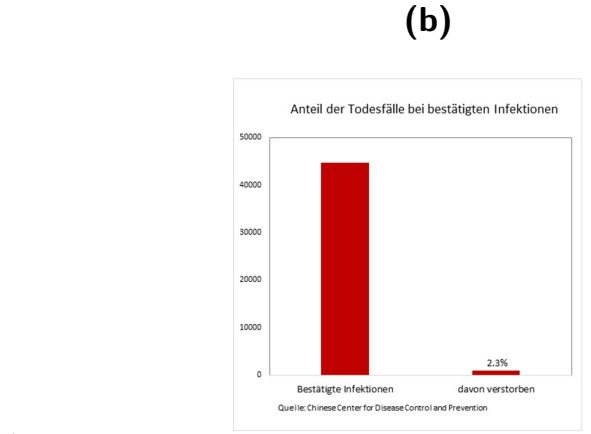

1

(c)

(d)

Figure 2: Information provided in Condition Average 
Condition Age Figure 3 displays the information given to the respondents assigned to Condition Age. This group of respondents receive information about the fact that the estimated case fatality rate of COVID-19 in China increases with age.



(a)

(d)

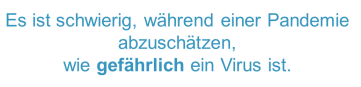

(b)

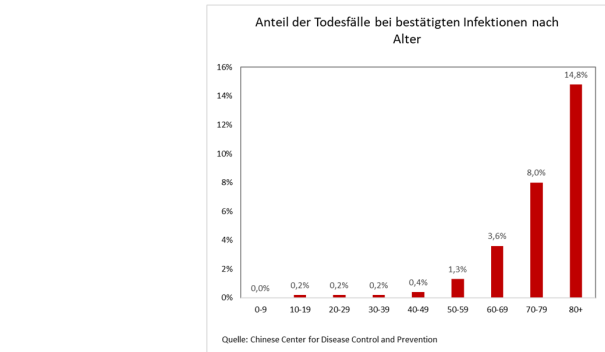

Eine Studie für China zeigt, dass die Gefährlichkeit des Virus mit steigendem Alter dramatisch zunimmt. (c)

(e)
Figure 3: Information provided in Condition Age

\section{Empirical strategy}

\subsection{Variables}

\subsubsection{Outcome variables}

We are interested in the effect of the restrictive measures on subjective well-being. Thus, we elicit how participants' subjective well-being has changed from the beginning of the year until now. Specifically, we ask them first how happy they were at the beginning of the year (on a scale from 1 to 10) and later in the survey we ask them to rate their current well-being. Our outcome variable of interest is then the difference between the two ratings.

Another main outcome of interest is participants' (hypothetical) willingness to pay for a discontinuation of the measures. We list the main restrictive measures imposed by the government in March and ask them to rank them in the order of importance by 
which they would like to discontinue them. Then we elicit participants' willingness to pay for the abolishment of the measure they ranked first. Afterwards we also elicit their willingness to pay for a discontinuation of all measures. The willingness to pay is always elicited in the form of a monthly payment over the next twelve months. Finally, we are interested is the relationship between general support for the restrictive measures and their continuation and our information conditions. To this end, we ask participants whether they think the measures are too radical/too weak and whether they want the measures to be continued for another four weeks after April 20. We also ask them whether they are happy to sacrifice some of their personal freedom to combat the spread of the virus.

\subsubsection{Covariates}

We elicit respondents' beliefs about case fatality rates (average, young and old) at the end of the survey. This way, we see (average) prior beliefs via the Control group, and we can see how well our information treatments worked (manipulation check) via the respondents in Condition Average and Condition Age.

Furthermore, we elicit in which state and Landkreis respondents live, whether they and their parents were born in Germany, their age, gender education, household income, household size, employment status and which sector they work in.

We also ask questions to elicit locus of control and psycholocical reactance. Furthermore, we ask several questions concerning respondents' current situation and how they deal with and are affected by the restrictive measures (financial worries, boredom, family frictions,..). We also ask about participants' living conditions (flat size, availability of outdoor space,...).

\subsection{Sample description}

We aim at targeting 4000 respondents from a representative sample of the German population (representative with respect to gender, age, income and region), which amounts to 1333 individuals per information condition in the survey. The panel, the distribution of the survey and the payments are administered by the survey company Respondi. ${ }^{2}$

\footnotetext{
${ }^{2}$ https://www.respondi.com/EN/
} 


\subsection{Empirical Analysis and main hypotheses}

We want to explore the data collected in our survey in several directions. First, we are interested in how people's subjective well-being is affected by the restrictive measures. Our outcome variable of interest in this part of the study is the difference between the assessed subjective well-being at the beginning of the year and the assessed subjective well-being now. We generally expect that this difference is negative, i.e. that the Corona crisis and the restrictive measures have reduced subjective wellbeing on average. We want to analyze how this difference is affected by individuals' psychological reactance. This personality trait is measured by a battery of questions that can be condensed into an index, quantifying how negatively an individual reacts to the imposition of restrictions and rules in general. We hypothesize that a clearer effect on the dislocation of subjective well-being from its normal long-run level should become apparent in persons with high psychological reactance.

Another focus of interest is participants' willingness to pay for the discontinuation of the restrictive measures in total, and also which measure they would like to abolish first and their willingness to pay for the discontinuation of this measure. We want to analyze how participants' willingness to pay to abolish the measures varies with age, income, job security and living situations, and also with personal character traits such as locus of control and psychological reactance (in the Control group).

Finally, we are interested in the relationship between the information conditions provided and the approval of measures, the willingness to continue the measures, the willingness to sacrifice personal freedom to reduce the spreading of the virus and the willingness to pay for a discontinuation of all measures. If there is a (latent) generational conflict arising from the introduction of the restrictive measures, we expect it to become more salient in Condition Age. We thus hypothesize that the willingness to continue the measures, the approval of the measures and the willingness to sacrifice personal freedom to combat the virus increase (more) with age in Condition Age compared to Condition Average and Condition Neutral. We expect the opposite pattern for respondents' willingness to pay to abolish all the measures. ${ }^{3}$

Furthermore, we expect that the effects we find will vary with individuals' incomes. We hypothesize that the measures hurt poor people more than rich people, for in-

\footnotetext{
${ }^{3}$ Of course, the direction of the effect in Condition Age will only be as expected if the information treatment increases peoples' perception of a generational conflict, either via a simple increase in salience (priming) or via additionally informing them about the risk/age differential. If people overestimate how much more dangerous COVID-19 is for old people compared to the young, our information treatment works in the opposite direction and decrease the perceived generational conflict. We would be able to detect this (on average) by looking at beliefs about case fatality rates in the Control group.
} 
stance because they are hit economically (losing their job,...) without having financial reserves, and also because they have to self-isolate in smaller flats, often without outdoor spaces, which can prove emotionally challenging. The difference in subjective well-being should thus be higher for respondents with relatively low incomes, and the willingness to continue the measures and the approval of measures should be positively correlated with income.

\subsection{Heterogeneity}

We will study heterogeneity using the background information on our respondents collected in the survey. Apart from heterogeneities with respect to age and income (which are already mentioned in our hypotheses), we will explore heterogenities with respect to gender and education. Furthermore, we will analyze how our effects vary with a battery of elicited control variables about personal circumstances and living situations (type of employment, flat size, personal health risks or family health risks,..). 